\title{
Prognostic value of tumor solid-part size and solid-part volume in patients with clinical stage I non-small cell lung cancer
}

\author{
Yoshihisa Shimada ${ }^{1}$, Hideyuki Furumoto ${ }^{1}$, Kentaro Imai ${ }^{1}$, Ryuhei Masuno ${ }^{2}$, Jun Matsubayashi ${ }^{3}$, \\ Naohiro Kajiwara ${ }^{1}$, Tatsuo Ohira ${ }^{1}$, Norihiko Ikeda ${ }^{1}$ \\ ${ }^{1}$ Department of Thoracic Surgery, ${ }^{2}$ Department of Radiology, ${ }^{3}$ Department of Anatomic Pathology, Tokyo Medical University, Tokyo, Japan \\ Contributions: (I) Conception and design: Y Shimada; (II) Administrative support: N Ikeda; (III) Provision of study materials or patients: Y Shimada, \\ H Furumoto, K Imai, R Masuno, J Matsubayashi; (IV) Collection and assembly of data: Y Shimada, H Furumoto, K Imai; (V) Data analysis and \\ interpretation: Y Shimada; (VI) Manuscript writing: All authors; (VII) Final approval of manuscript: All authors. \\ Correspondence to: Yoshihisa Shimada, MD, PhD. Department of Thoracic Surgery, Tokyo Medical University, 6-7-1 Nishishinjuku, Shinjuku-ku, \\ Tokyo 160-0023, Japan. Email: zenkyu@za3.so-net.ne.jp.
}

Background: This study aimed to predict the malignant potential of clinical stage I non-small cell lung cancer (c-I NSCLC) by semiautomatic three-dimensional (3D) volumetric measurement of a tumor (3D-data) and the axial computed tomography (CT) data derived from a 3D volumetric dataset (2D-data). The predictive performance was evaluated in terms of overall survival (OS), disease-free survival (DFS), and pathological invasive factors (positive lymphatic invasion, blood vessel invasion, pleural invasion, or lymph node metastasis).

Methods: We identified 252 patients (122 male; mean age, 68 years; range, 23-84 years) with c-I NSCLC who underwent high resolution CT and reconstruction of 3D imaging, followed by complete resection between January 2012 and December 2015. In this study, 2D-data including whole tumor size (WTS) and solid-part size (SPS) and 3D-data including whole tumor volume (WTV) and solid-part volume (SPV) acquired by a $3 \mathrm{D}$ volume rendering software were analyzed.

Results: The area under the receiver operating characteristic (ROC) curve for WTS, SPS, WTV, SPV relevant to recurrence was $0.667,0.727,0.654$, and 0.751 while analyses of ROC curves revealed optimal WTS, SPS, WTV, and SPV cut-off values to predict recurrence of $2.48 \mathrm{~cm}, 2.03 \mathrm{~cm}, 3,258 \mathrm{~mm}^{3}$ and $1,889 \mathrm{~mm}^{3}$, respectively. The association between SPS and SPV was the coefficient of determination $\left(R^{2}\right)=0.59$. Multivariate analysis showed that $\mathrm{SPV}>1,889 \mathrm{~mm}^{3}(\mathrm{P}=0.016)$ and male $(\mathrm{P}=0.041)$ were significant predictors of $\mathrm{OS}$ whereas $\mathrm{SPV}>1,889 \mathrm{~mm}^{3}(\mathrm{P}=0.001)$, male $(\mathrm{P}=0.003)$, and the serum carcinoembryonic antigen value $(\mathrm{P}=0.041)$ were significantly correlated with $\mathrm{DFS}$. SPS, SPV as well as the combination of SPS and SPV were all significantly correlated with the prediction of OS and DFS, and the incidence of pathological invasive factors.

Conclusions: SPV and the integrated use of SPS and SPV was highly beneficial for the prediction of postoperative prognosis in c-I NSCLC.

Keywords: Non-small cell lung cancer (NSCLC); volume; solid-part; three-dimensional CT (3DCT); stage I

Submitted Mar 09, 2018. Accepted for publication Oct 11, 2018.

doi: $10.21037 /$ jtd.2018.11.08

View this article at: http://dx.doi.org/10.21037/jtd.2018.11.08

\section{Introduction}

Recent advances in imaging technology in the field of lung cancer have enabled the stratification of peripheral nonsmall cell lung cancer (NSCLC) patients according to their prognosis by evaluating the high-resolution computed tomography (HRCT) features of their lung tumor (1-5). The ratio of the maximum consolidation diameter to the maximum tumor diameter is widely accepted for predicting 
the outcome of patients who underwent not only pulmonary resection but also stereotactic body radiotherapy $(4,6)$. Patients with a smaller proportion of solid-part size in lung cancer on HRCT had a significantly lower recurrence rate regardless of the operative procedures, suggesting that the separate measurements of the solid-part size and the whole tumor size are highly beneficial for tailored treatment planning $(2,4)$. Although decreased CT-measured solid-part size of lung cancer, especially adenocarcinoma, is reported to be a better prognostic factor, small lung lesions with an irregularly shaped solid part in the greatest dimension are often encountered and can cause intraobserver and interobserver variability in practice.

To address the issue, we preoperatively take advantage of three-dimensional (3D) visualization using volume rendered imaging technique (3D-VRT) not only for surgical simulation but also for possible prediction of the pathobiological features of a lung tumor and prognostic outcomes by conducting volumetric quantification of the solid and non-solid parts separately using a 3DCT workstation (Fujifilm Synapse Vincent system; Fujifilm Corp., Tokyo, Japan) (7-11). Semiautomatic volumetric measurement of a tumor presents a pixel-wise segmentation of the 3DCT volume depending on the attenuation of each single voxel. As the calculation of the solid-part and non-solid-part volume based on 3D-VRT is consistent, the volumetric quantification is more likely to reflect the patient's prognosis.

This study aimed to investigate the clinical outcomes and pathological malignant grade of patients with resected clinical stage I NSCLC by comparing preoperative HRCT and 3D-VRT oriented radiologic parameters such as solidpart size and solid-part volume.

\section{Methods}

\section{Patients}

We identified 252 patients with clinical stage I NSCLC who underwent complete resection between January 2012 and December 2015 at our department. HRCT and 3DCT were preoperatively performed for all patients using the Fujifilm Synapse Vincent system. The Tumor Node Metastasis (TNM) stage was determined in accordance with the $8^{\text {th }}$ edition of the TNM classification of malignant tumors (12). Preoperative evaluation included physical examination, chest radiography, chest and abdomen CT, blood examination, and F-18 fluorodeoxyglucose-positron emission tomography/computed tomography (FDG-PET/ CT). The protocols for data collection and analysis were approved and the need to obtain written informed consent from each patient was waived by the Institutional Review Board of Tokyo Medical University.

\section{HRCT and 3DCT evaluation for primary tumors}

The patients underwent contrast-enhanced CT imaging using a 64-channel multidetector CT system (Light Speed VCT, GE Medical systems, Milwaukee, WI, USA). Axial sections (1.25 mm in thickness) of the whole lung were reconstructed at intervals of $1.0 \mathrm{~mm}$, and images were viewed on standard lung windows (level, $-600 \mathrm{HU}$; width $1,500 \mathrm{HU}$ ) with the sharp kernel of the version "LUNG" and mediastinal windows (level, $30 \mathrm{HU}$; width $400 \mathrm{HU}$ ). Each CT image was acquired within 1 breath hold of about $5 \mathrm{~s}$, after a delay of $70 \mathrm{~s}$ during which the contrast media injection took effect.

HRCT scans were evaluated by at least 2 surgeons (Y Shimada, H Furumoto, K Imai) and 1 radiologist (R Masuno) in consensus. We defined the soli-part size as the maximum dimension of the solid component of the lung windows excluding non-solid-part and measured the maximum diameter of tumor on axial CT slices (Figure 1). Non-solid-part representing ground glass opacity was defined as a misty increase in lung attenuation that did not obscure the underlying vascular markings.

The presented CT scan protocol has been used for both standard staging for lung cancer patients amenable to contrast radiography and $3 \mathrm{D}$ image conversion using the Synapse Vincent system. After digital imaging and communication in medicine data were transferred to a workstation using the indicated software, 3D-VRT were performed (Figure 1). In terms of volumetric measurement of a lung tumor, a density level of $-300 \mathrm{HU}$ is the threshold level that separate solid-part from non-solid-part by the software algorithm. This $3 \mathrm{D}$ tumor analysis provided the whole tumor volume, solid-part volume, and non-solid-part volume separately. The semiautomated volumetric analysis performed by the software needed to be adjusted manually. The results were confirmed in consensus ( 2 or 3 surgeons, Y Shimada, H Furumoto, K Imai, 1 radiologist, R Masuno).

\section{Definition of pathological invasive factors and patient outcomes}

The available pathology reports for all the 252 surgical 

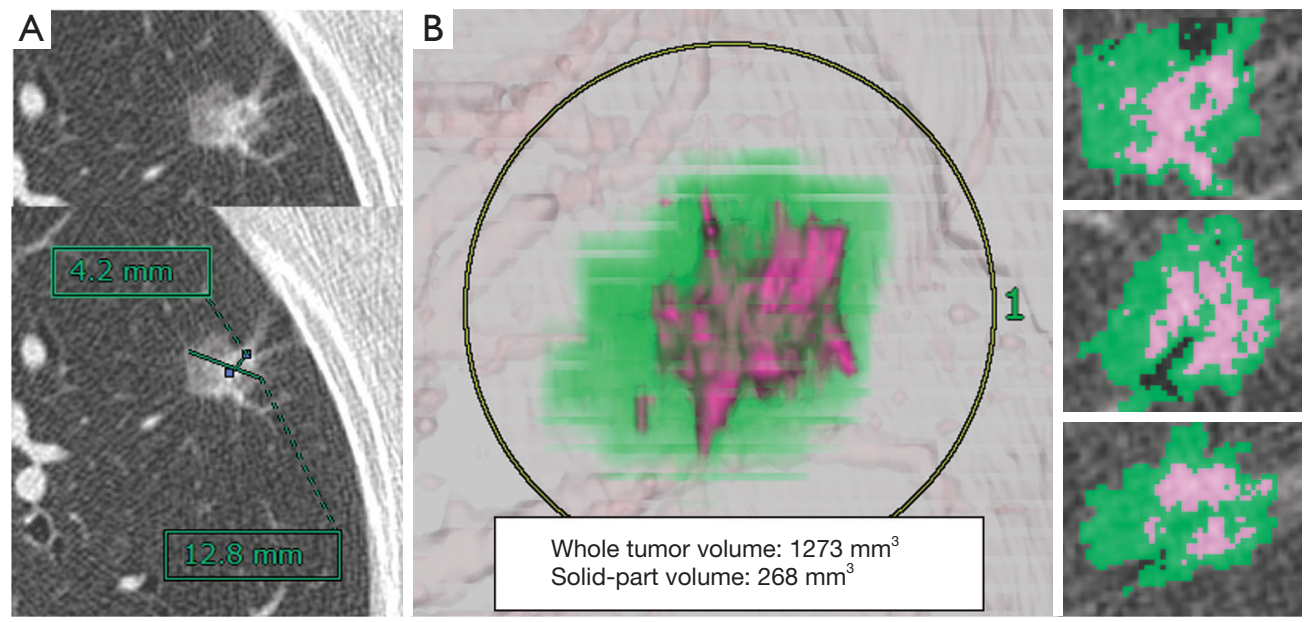

Figure 1 Representative comparative images of a lesion's whole tumor size and solid-part size on high-resolution computed tomography (HRCT), and whole tumor volume and solid-part volume measured by the Synapse Vincent system. An HRCT image presenting whole tumor size of $1.28 \mathrm{~cm}$ and solid-part size of $0.42 \mathrm{~cm}(\mathrm{~A})$, and a three-dimensional (3D) volume rendered images of a tumor presenting whole tumor volume of $1,273 \mathrm{~mm}^{3}$ and solid-part volume of $268 \mathrm{~mm}^{3}$ with serial sections of the display (B). The 3D volume rendered images result in the detection of a solid-part with red and a ground glass opacity part with green.

specimens were reviewed in this study. Blood vessel invasion $(V)$ was identified by the presence of erythrocytes in the lumen or an endothelial cell lining, or the presence of elastic tissue around large vessels while lymphatic invasion (Ly) was determined to be present when tumor cells floating in lymphatic vessels with no supporting smooth muscles or elastic fibers were identified. Resected lymph nodes were sectioned thicker than $2 \mathrm{~mm}$ and correctly embedded to ensure that the pathologists can identify all macrometastases. At least single or 2 sections from blocks were prepared for diagnosis. Pathological invasive factors were defined as positive Ly, $\mathrm{V}$, pleural invasion (PL), or lymph node metastasis. Overall survival (OS) was measured from the date of surgery to the date of death from any cause or the date on which the patients were last known to be alive. Disease-free survival (DFS) was measured from the date of surgery until the first event (relapse or death from any cause or the last follow-up visit).

\section{Statistical analysis}

The receiver operating characteristic (ROC) curves of each parameter were used to predict recurrence. The area under the ROC curve (AUC) with its $95 \%$ confidence interval (CI) was measured. OS and DFS curves were plotted using the Kaplan-Meier method, and differences in variables were determined using the log-rank test. Categorical comparison was performed using the Pearson chi-squared test for discrete data and Student $t$-test for continuous data. Pearson correlation coefficient was used to determine the correlation between solid-part size and solid-part volume and the value of the coefficient of determination $\left(R^{2}\right)$ was also provided. HRCT and 3DCT parameters measured by three observers were recorded, and interobserver variability by using a random sample of 100 patients was calculated for intraclass correlation coefficient (ICC). Univariate analysis for OS and DFS was performed using the Cox proportional hazards regression model whereas multivariate analysis was conducted with a stepwise variable selection for the Cox model. All tests were two-sided, and $\mathrm{P}$ values of less than 0.05 were considered to indicate a statistically significant difference. SPSS statistical software package (version 24.0; DDR3 RDIMM, SPSS Inc., Chicago, IL, USA) was used for statistical analysis.

\section{Results}

The characteristics of the patients were shown in Table 1 . The median follow-up time for survivors was 1,233 days (range, 16 to 2,227 days). During the study period, recurrences occurred in 29 patients (12\%), some of whom eventually died from these relapses $(n=12,5 \%)$. Only 1 patient died from subarachnoid hemorrhage during the study period. The clinical TNM stage showed 186 patients 
Table 1 Patient characteristics

\begin{tabular}{|c|c|}
\hline Variable & Value $(n=252)(\%)$ \\
\hline Age, mean [range] (years) & 68 [23-84] \\
\hline \multicolumn{2}{|l|}{ Sex } \\
\hline Male & $122[48]$ \\
\hline Female & $130[52]$ \\
\hline \multicolumn{2}{|l|}{ cTNM stage } \\
\hline Stage IA & $186[74]$ \\
\hline Stage IB & 66 [26] \\
\hline \multicolumn{2}{|l|}{ Lymphatic invasion } \\
\hline Positive & 83 [33] \\
\hline Negative & 169 [67] \\
\hline \multicolumn{2}{|l|}{ Blood vessel invasion } \\
\hline Positive & $81[32]$ \\
\hline Negative & $171[68]$ \\
\hline \multicolumn{2}{|l|}{ Pleural invasion } \\
\hline Positive & 48 [19] \\
\hline Negative & 204 [81] \\
\hline \multicolumn{2}{|l|}{ Lymph node metastasis } \\
\hline Positive & 34 [13] \\
\hline Negative & 218 [87] \\
\hline \multicolumn{2}{|l|}{ Histology } \\
\hline Adenocarcinoma & $221[88]$ \\
\hline Non-adenocarcinoma & 31 [12] \\
\hline $\begin{array}{l}\text { Whole tumor size, } \\
\text { range (mean } \pm \mathrm{SD})(\mathrm{cm})\end{array}$ & $0.50-4.00(2.23 \pm 0.75)$ \\
\hline $\begin{array}{l}\text { Solid-part size, } \\
\text { range (mean } \pm \text { SD) }(\mathrm{cm})\end{array}$ & $0-3.91(1.67 \pm 0.99)$ \\
\hline $\begin{array}{l}\text { Whole tumor volume, } \\
\text { range }(\text { mean } \pm \mathrm{SD})\left(\mathrm{mm}^{3}\right)\end{array}$ & $51-24,393(4,239 \pm 3,975)$ \\
\hline $\begin{array}{l}\text { Solid-part volume, } \\
\text { range }(\text { mean } \pm \mathrm{SD})\left(\mathrm{mm}^{3}\right)\end{array}$ & $0.4-16,344(2,350 \pm 2,761)$ \\
\hline
\end{tabular}

TNM, pathological tumor, node and metastasis; SD, standard deviation.

(74\%) with stage IA and 66 patients (26\%) with stage IB. The mean whole tumor size, solid-part size, whole tumor volume, and solid-part volume were $2.23 \pm 0.75 \mathrm{~cm}$, $1.67 \pm 0.99 \mathrm{~cm}, 4,239 \pm 3,975 \mathrm{~mm}^{3}$, and 2,350 $\pm 2,761 \mathrm{~mm}^{3}$ while the median whole tumor size, solid-part size, whole

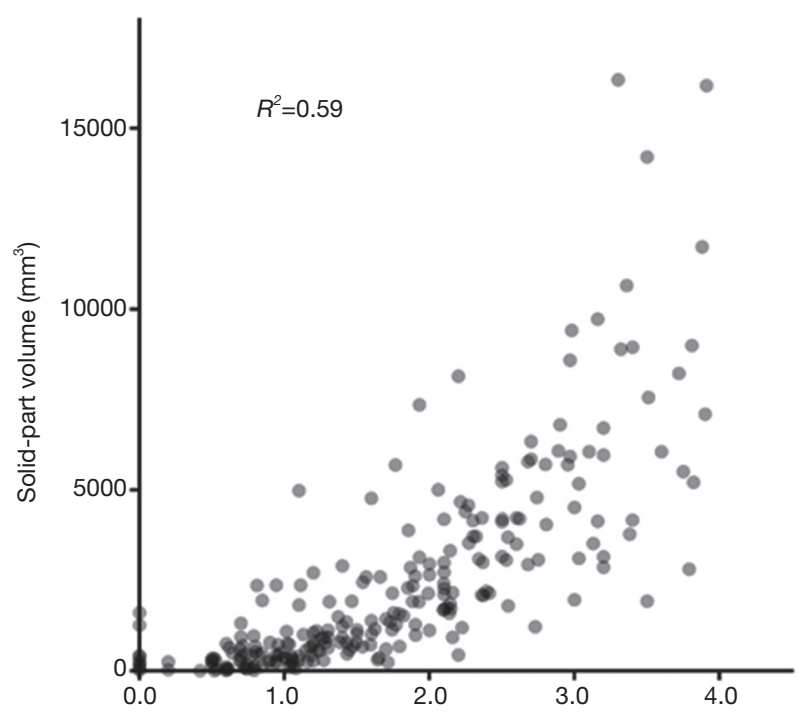

Figure 2 Correlation between solid-part size and solid-part volume as determined using Pearson correlation coefficient $\left(R^{2}=0.59\right)$.

tumor volume, and solid-part volume were $2.18 \mathrm{~cm}$, $1.56 \mathrm{~cm}, 3,063 \mathrm{~mm}^{3}$ and $1,275 \mathrm{~mm}^{3}$, respectively. The pathological findings indicated that the numbers of patients with Ly, V, PL, and lymph node metastasis were 83 (33\%), 81 (32\%), 48 (19\%), and 34 (13\%), respectively.

We analyzed the correlation between solid-part size and solid-part volume using Pearson correlation coefficient $\left(R^{2}=0.59\right.$; Figure 2$)$, and this coefficient value showed a strong correlation. Interobserver agreements for volumetric measurement of a tumor by the dedicated software (ICC $=0.977$ ) was higher than the measurement of solidtumor size on HRCT (ICC $=0.925$, Table 2).

To investigate the impact of HRCT and 3D volumetric parameters for prognostic outcomes, we analyzed the ROC curves for recurrences (Figure 3). AUC and the optimal cut-off value relevant to recurrence for whole tumor size, solid-part size, whole tumor volume, and solid-part volume were 0.667 and $2.48 \mathrm{~cm}$ (Figure $3 A$ ), 0.727 and $2.03 \mathrm{~cm}$ (Figure 3B), 0.654 and $3,258 \mathrm{~mm}^{3}$ (Figure 3C), and 0.751 and $1,889 \mathrm{~mm}^{3}$ (Figure $3 D$ ), respectively. Univariate and multivariate analyses were performed to examine the association between OS or DFS and clinical factors including HRCT and 3D volumetric radiologic features (Tables 3,4). Univariate analysis identified the following as significant predictors of unfavorable OS: age $(\mathrm{P}=0.032)$, male $(\mathrm{P}=0.019)$, the presence of smoking history $(\mathrm{P}=0.037)$, whole tumor volume $>3,258 \mathrm{~mm}^{3}(\mathrm{P}=0.044)$, and solidpart volume $>1,889 \mathrm{~mm}^{3}(\mathrm{P}=0.008)$. Multivariate analysis 
Table 2 Analysis of interobserver reproducibility of solid-tumor size and solid-tumor volume

\begin{tabular}{lccc}
\hline Parameter & Patients, $\mathrm{n}$ & ICC $(95 \% \mathrm{Cl})$ & $\mathrm{P}$ value \\
\hline Solid-tumor size on HRCT & 100 & $0.925(0.881-0.956)$ & $0.977(0.959-0.987)$ \\
Solid-tumor volume by volumetric measurement with 3D-VRT & 100 & $<0.001$ & $<0.001$ \\
\hline
\end{tabular}

ICC, intra-class correlation coefficient; Cl, confidence interval; HRCT, high-resolution computed tomography; 3D-VRT, three-dimensional volume rendered imaging techniques.
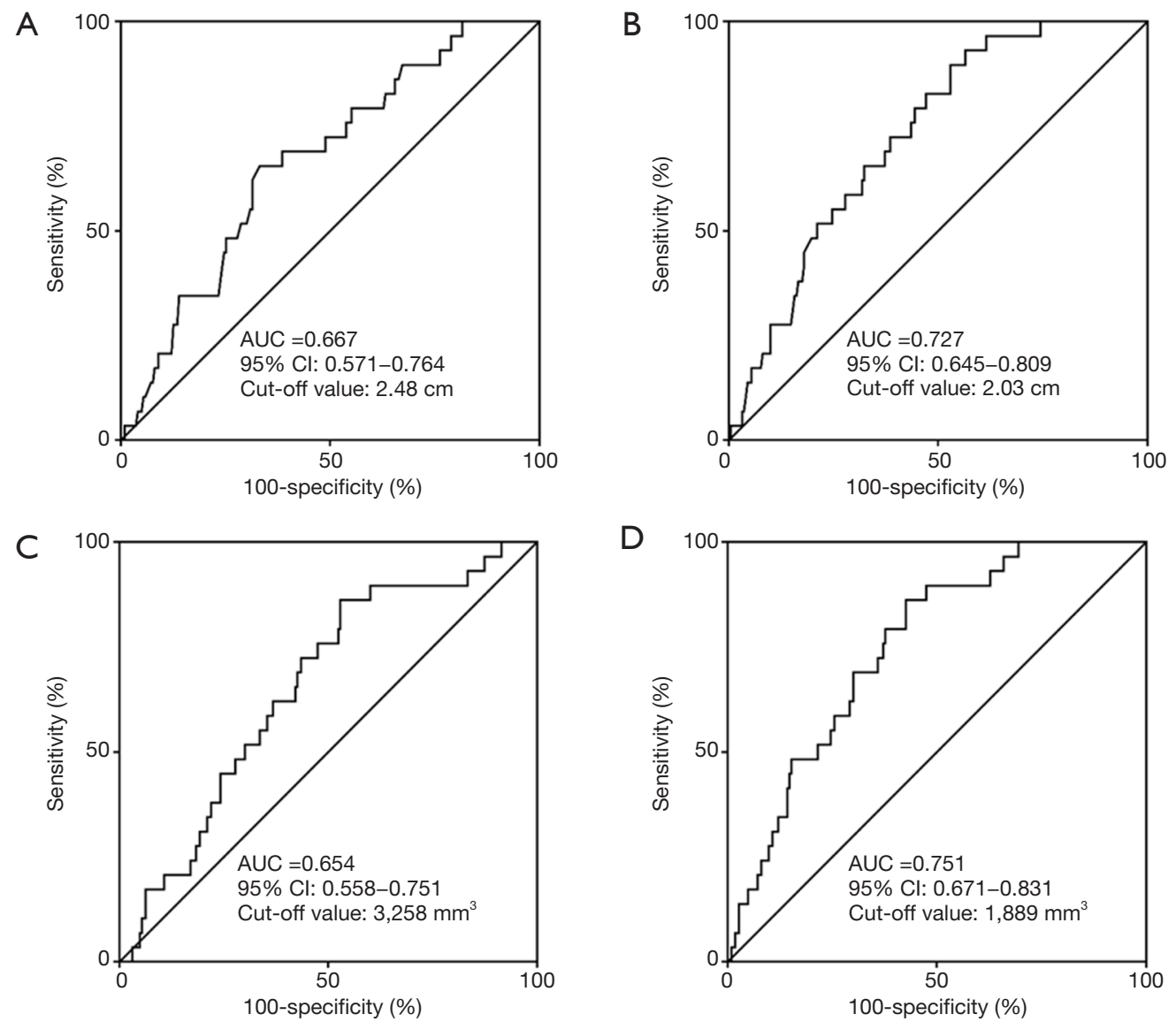

Figure 3 The area under the receiver operating characteristic (ROC) curve for recurrence as determined using whole tumor size (A), soli-part size (B), whole tumor volume (C), and solid-part volume (D) in patients with stage I non-small cell lung cancer patients. AUC, area under the curve; CI, confidence interval.

revealed the following as independently associated with OS (Table 3): sex $(\mathrm{P}=0.041)$ and solid-part volume $>1,889 \mathrm{~mm}^{3}$ $(\mathrm{P}=0.016)$. Univariate analysis identified the following as significant predictors of unfavorable DFS: sex $(\mathrm{P}=0.001)$, the presence of smoking history $(\mathrm{P}=0.001)$, the serum carcinoembryonic antigen (CEA) value $(\mathrm{P}=0.001)$, stage IB $(\mathrm{P}=0.027)$, whole tumor size $>2.48 \mathrm{~cm}(\mathrm{P}=0.001)$, solid-part size $>2.03 \mathrm{~cm}(\mathrm{P}<0.001)$, whole tumor volume
$>3,258 \mathrm{~mm}^{3}(\mathrm{P}=0.005)$, and solid-part volume $>1,889 \mathrm{~mm}^{3}$ $(\mathrm{P}<0.001)$. Multivariate analysis revealed the following as independently associated with DFS (Table 4): sex $(\mathrm{P}=0.003)$, the serum CEA level $(\mathrm{P}=0.041)$, and solid-part volume $>1,889 \mathrm{~mm}^{3}(\mathrm{P}=0.001)$.

We further assessed whether the integrated use of both solid-part size and solid-part volume was beneficial for the prediction of postoperative prognosis. Patients were 
Table 3 Univariate and multivariate analyses for overall survival

\begin{tabular}{|c|c|c|c|c|}
\hline Variable & \multicolumn{2}{|c|}{ Univariate analysis } & \multicolumn{2}{|c|}{ Multivariate analysis } \\
\hline Age & $1.083(1.007-1.166)$ & 0.032 & - & - \\
\hline Sex (male vs. female) & $6.060(1.340-27.027)$ & 0.019 & $4.852(1.069-22.016)$ & 0.041 \\
\hline Smoking history (yes vs. no) & $4.953(1.098-22.349)$ & 0.037 & - & - \\
\hline cTNM stage (IB vs. IA) & $1.255(0.386-4.079)$ & 0.705 & - & - \\
\hline Whole tumor size (>2.48 vs. $\leq 2.48 \mathrm{~cm}$ ) & $2.730(0.893-8.347)$ & 0.078 & - & - \\
\hline Solid-part size (>2.03 vs. $\leq 2.03 \mathrm{~cm}$ ) & $2.907(0.951-8.889)$ & 0.061 & - & - \\
\hline Whole tumor volume $\left(>3,258 \mathrm{vs} . \leq 3,258 \mathrm{~mm}^{3}\right)$ & $3.756(1.033-13.548)$ & 0.044 & - & - \\
\hline
\end{tabular}

Table 4 Univariate and multivariate analyses for disease-free survival

\begin{tabular}{|c|c|c|c|c|}
\hline Variable & \multicolumn{2}{|c|}{ Univariate analysis } & \multicolumn{2}{|c|}{ Multivariate analysis } \\
\hline Age & $1.030(0.990-1.071)$ & 0.141 & - & - \\
\hline Sex (male vs. female) & $4.632(1.893-11.332)$ & 0.001 & $3.965(1.614-9.740)$ & 0.003 \\
\hline Smoking history (yes vs. no) & $4.739(1.815-12.346)$ & 0.001 & - & - \\
\hline cTNM stage (IB vs. IA) & $2.237(1.086-4.608)$ & 0.027 & - & - \\
\hline Whole tumor size (>2.48 vs. $\leq 2.48 \mathrm{~cm})$ & $3.571(1.669-7.634)$ & 0.001 & - & - \\
\hline Solid-part size (>2.03 vs. $\leq 2.03 \mathrm{~cm}$ ) & $3.861(1.805-8.264)$ & $<0.001$ & - & - \\
\hline Whole tumor volume $\left(>3,258 \mathrm{vs} . \leq 3,258 \mathrm{~mm}^{3}\right)$ & $3.226(1.435-7.246)$ & 0.005 & - & - \\
\hline
\end{tabular}

$\mathrm{Cl}$, confidence interval; CEA, carcinoembryonic antigen; TNM, pathological tumor, node and metastasis.

divided into three groups; group A, patients with solid-part size $\leq 2.03 \mathrm{~cm}$ and solid-part volume $\leq 1,889 \mathrm{~mm}^{3}$; group $\mathrm{B}$, patients with either solid-part size $>2.03 \mathrm{~cm}$ or solidpart volume $>1,889 \mathrm{~mm}^{3}$; group $\mathrm{C}$, patients with solidpart size $>2.03 \mathrm{~cm}$ and solid-part volume $>1,889 \mathrm{~mm}^{3}$. The predictive performance of the pathological invasive factors, and OS and DFS outcomes by using the cut-off values were demonstrated in Table 5. Both solid-part size $(\mathrm{P}<0.001)$ and solid-part volume $(\mathrm{P}<0.001)$ showed a significant correlation with the incidence of pathological invasive factors. Group C also exhibited high proportion of invasive factors compared to Group A $(\mathrm{P}<0.001)$. The Kaplan-Meier curves for OS and DFS (Figure 4) showed that solid-part size influenced
OS $(\mathrm{P}=0.050$; Figure $4 A)$ and DFS $(\mathrm{P}<0.001$; Figure $4 D)$ whereas solid-part volume was also significantly correlated with OS $(\mathrm{P}=0.002$; Figure $4 B)$ and DFS $(\mathrm{P}<0.001$; Figure $4 E)$. Subgroup analysis with the combination revealed that the 4-year OSs of $96.6 \%, 91.2 \%$, and $87.4 \%$ and the 4 -year DFSs of $93.4 \%, 88.4 \%$, and $73.4 \%$ for group A, B, and C, respectively (Figure 4C,F and Table 5). The differences in OS were statistically significant between group A and $\mathrm{C}(\mathrm{P}=0.005)$, and between group $\mathrm{A}$ and $\mathrm{B}(\mathrm{P}=0.018)$. The differences in DFS between group A and C was statistically significant $(\mathrm{P}<0.001)$ while that between group $\mathrm{A}$ and $\mathrm{B}$ was marginally significant $(\mathrm{P}=0.093)$. Together, group $\mathrm{C}$ had the most unfavorable OS and DFS compared to group A, B as 
Table 5 Prognostic impact of preoperative computed tomographic imaging factors

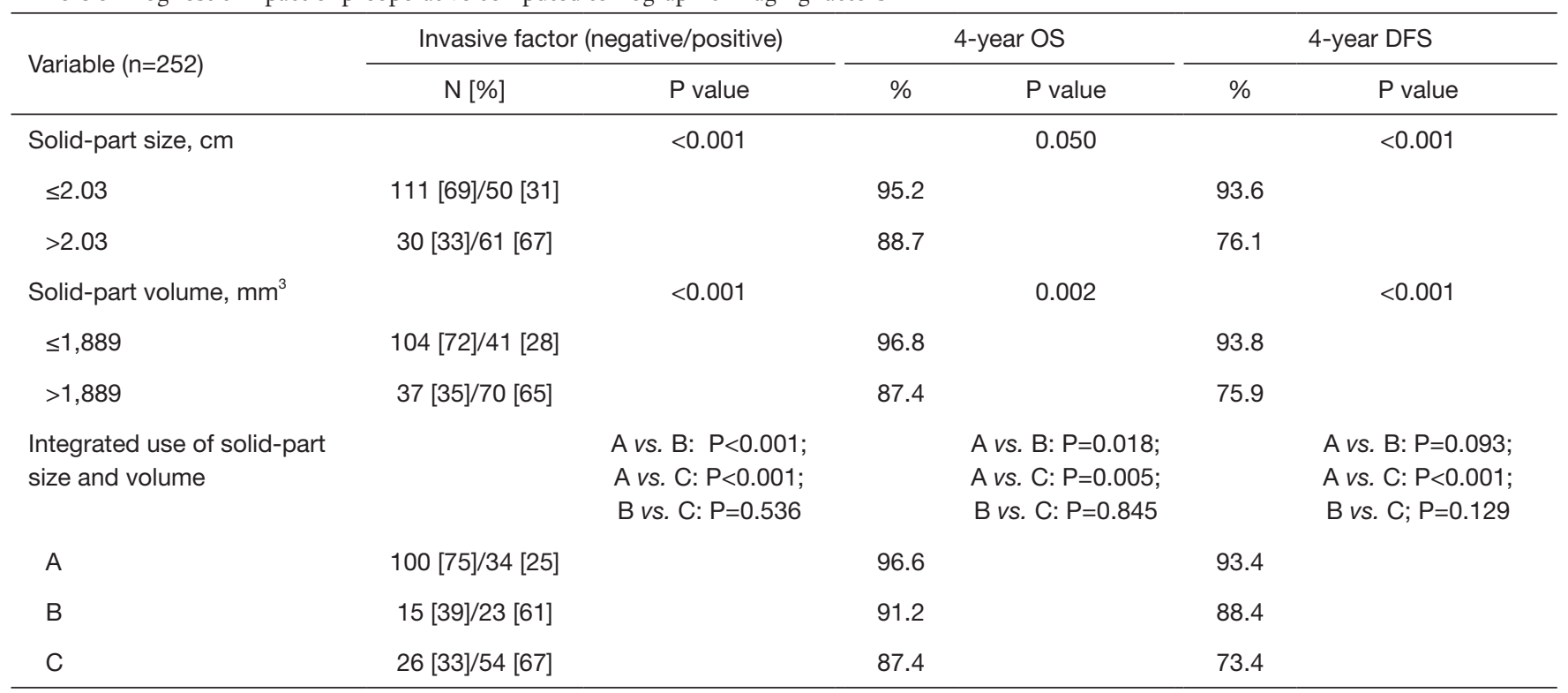

Invasive factor: positive lymphatic invasion, vascular invasion, pleural invasion, or lymph node metastasis. OS, overall survival; DFS, disease-free survival; $A$, patients with solid-part size $\leq 2.03 \mathrm{~cm}$ and solid-part volume $\leq 1,889 \mathrm{~mm}^{3}$; B, patients with either solid-part size $>2.03 \mathrm{~cm}$ or solid-part volume $>1,889 \mathrm{~mm}^{3}$; C, patients with solid-part size $>2.03 \mathrm{~cm}$ and solid-part volume $>1,889 \mathrm{~mm}^{3}$.

well as individual use of either solid-part size or sold-part volume.

\section{Discussion}

In this study, we evaluated the survival outcomes and association between CT variables and pathological findings by comparing the predictive performance of whole tumor size, solid-part size, whole tumor volume, and solidpart volume in patients with clinical stage I NSCLC. Multivariate analyses for the prediction of unfavorable OS and DFS showed that solid-part volume had a significant correlation with both outcomes. The combined use of solid-part size and solid-part volume also contributed to the excellent stratification of patients on prognosis.

The ability to preoperatively estimate survival outcomes and the presence of prognostic pathological factors on imaging modalities is crucial to the determination of the appropriate treatment strategy. Lymphatic invasion and blood vessel invasion in patients with NSCLC have been reported to be a strong independent predictor for recurrence (13-16). Preoperative prediction of pathological invasive factors in the current study including lymphatic invasion, blood vessel invasion, pleural invasion, and lymph node metastasis is of importance to select appropriate surgical procedures since all these factors are responsible for increased recurrence rate and unfavorable prognosis (12-17). A greater number of studies have shown a careful observation of HRCT-based features such as the calculation of consolidation to tumor ratio of lung cancer, and paying attention to the solid-part size of small peripheral lung cancer helps in predicting prognosis and pathological malignancy (2-4,18-20). At present, conducting HRCT before any treatment to evaluate small peripheral NSCLC is mandatory as the maximum diameter of the solid-part serves as a determinant for the latest TNM classification. Although those parameters are very important, it is occasionally difficult to even judge whether the lesion of interest is a predominantly solid or non-solid-predominant tumor because of the irregularity of the solid-part shape.

A $3 \mathrm{D}$ virtual lung surgery planning workstation provides surgeons with an effective tool for safe and rational surgery (7-9). In terms of its extensive role, 3D-VRT provides not only visual information for surgical simulations but also quantitative information such as the volume of the lungs and tumor. Takenaka et al. reported that the solidpart volume as quantified by the Synapse Vincent system was a prominent predictive factor for DFS compared with the whole tumor volume or solid-part size on HRCT (21). Comparative analysis of interobserver reproducibility of 
A

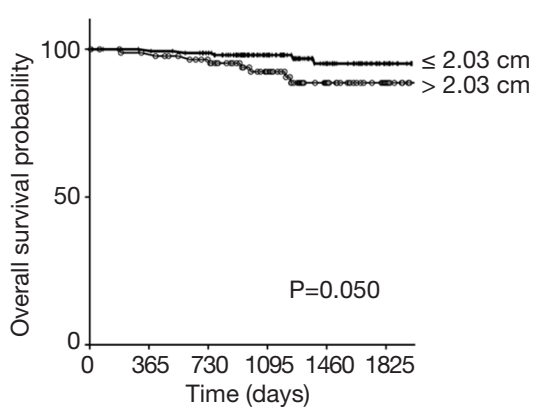

At risk

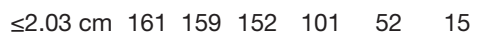

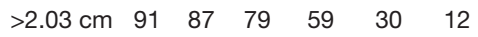

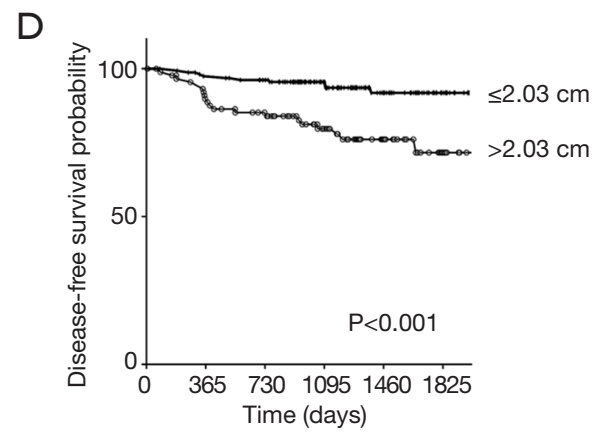

At risk

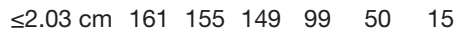

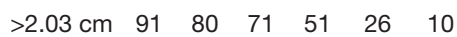

B

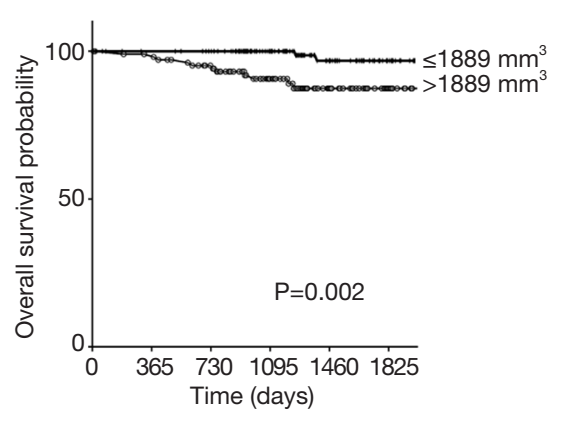

At risk

$\begin{array}{lllllll}\leq 1,889 \mathrm{~mm}^{3} & 145 & 142 & 137 & 94 & 48 & 15\end{array}$

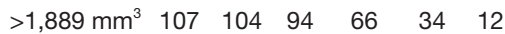

E

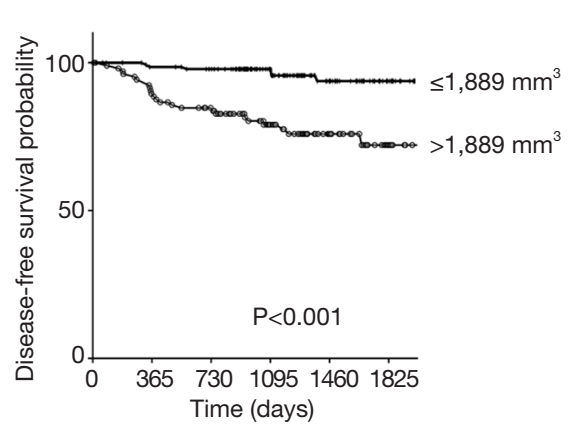

At risk

$\begin{array}{lllllll}\leq 1,889 \mathrm{~mm}^{3} & 145 & 140 & 135 & 92 & 46 & 15\end{array}$

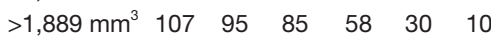

C
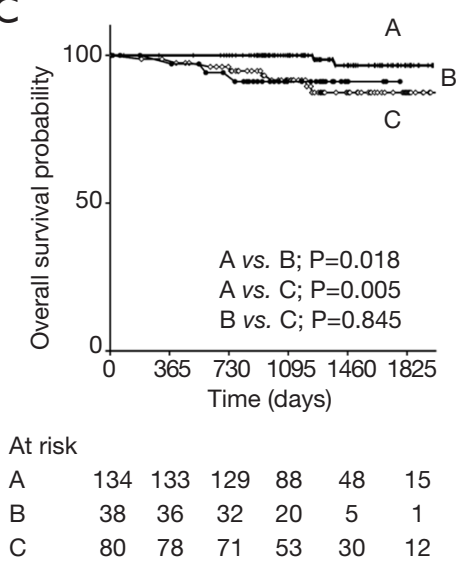

$\mathrm{F}$

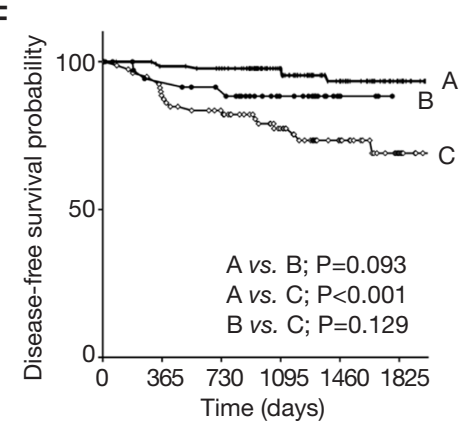

At risk

$\begin{array}{lcccccc}\text { A } & 134 & 131 & 127 & 86 & 46 & 15 \\ \text { B } & 38 & 34 & 31 & 20 & 5 & 1 \\ \text { C } & 80 & 71 & 63 & 45 & 26 & 10\end{array}$

Figure 4 Overall survival (OS) and disease-free survival (DFS) curves of patients with clinical stage I non-small cell lung cancer. All the patients were divided into three groups due to the cut off values of solid-part size and solid-part volume; group A, patients with solid-part size $\leq 2.03 \mathrm{~cm}$ and solid-part volume $\leq 1,889 \mathrm{~mm}^{3}$; group B, patients with either solid-part size $>2.03 \mathrm{~cm}$ or solid-part volume $>1,889 \mathrm{~mm}{ }^{3}$; group C, patients with solid-part size $>2.03 \mathrm{~cm}$ and solid-part volume $>1,889 \mathrm{~mm}^{3}$. (A,B) OS curves according to solid-part size (A) and solid-part volume (B) that were dichotomized at each cut-off value, $2.03 \mathrm{~cm}$ and $1,889 \mathrm{~mm}^{3}$; (C) OS curves according to the three groups. $(\mathrm{D}, \mathrm{E}, \mathrm{F})$ DFS curves according to solid-part size (D), solid-part volume (E), and the three groups (F).

solid-tumor size and volume (Table 2) revealed that the workstation semiautomatically provided reproducible and consistent volumetric information of the tumor of interest with ICC of 0.977. Even though the volumetric quantification of a tumor by $3 \mathrm{D}-\mathrm{VRT}$ is time consuming in contrast to tumor size measurements on conventional axial CT slices, the measurement of solid-part volume for estimating prognostic outcomes such as OS, DFS, and pathological invasive factors supports the successful planning of an appropriate surgical strategy and can contribute to individualized surgical approach including the indication of limited resection for stage I NSCLC. Since there was a strong correlation between solid-part size and solid-part volume in the current study (Figure 2), solidpart size and solid-part volume resulted in the confounding factors one another. That could be the reason why solidpart size was not independent predictive factors for OS and DFS on our multivariate analyses. The measurement of solid-part size on HRCT is supposed to be fundamental to determine $\mathrm{T}$ factor, and simpler and easier method to predict postoperative survival and recurrence. On the contrary, although surgical simulations by $3 \mathrm{D}-\mathrm{VRT}$ offer us to do better surgical planning and more deeply understand lung cancer pathobiology, this process can be time- and cost-intensive compared with the conducting HRCT alone. Therefore, the proposed application of this data to clinical 
scenarios of early-staged lung cancer unless 3D-VRT can be performed routinely would be to add volumetric quantification of lung tumor for a tailored surgical approach when the shape of the solid-part in greatest dimension on HRCT is too irregular and heterogenous to measure in consensus.

The limitations of this study are its retrospective nature and potential bias. The cut-off values of the ROC curves that dichotomized the two groups could be an arbitrarily value and not available universally. We measured tumor volume by initially connecting the end-to-end dimensions of a tumor with a straight line in an axial plane on the workstation, which was similar to the methodology on HRCT. However, once we delineate the end-to-end line, whole tumor volume, solid-part volume, and non-solid-part volume were separately generated semiautomatically with excellent interobserver agreement.

In conclusion, solid-part volume was significantly associated with the prediction of OS, DFS, and the presence of pathological invasive factors. The measurement of not only solid-part size but also solid-part volume is useful in patients with clinical stage I NSCLC when the measurement of the solid-part on HRCT is too complicated due to the chance of inconsistent or irregular-shaped findings.

\section{Acknowledgements}

The authors are indebted to the medical editors of the Department of International Medical Communications of Tokyo Medical University for editing the English manuscript. We also thank Ms. Mami Murakami for helping with the statistical analyses of this work.

\section{Footnote}

Conflicts of Interest: The authors have no conflicts of interest to declare.

Ethics Statement: This study was approved by the Institutional Ethics Board of Tokyo Medical University (No. 3907).

\section{References}

1. Takamochi K, Nagai K, Yoshida J, et al. The role of computed tomographic scanning in diagnosing mediastinal node involvement in non-small cell lung cancer. J Thorac
Cardiovasc Surg 2000;119:1135-40.

2. Tsutani $Y$, Miyata $Y$, Nakayama H, et al. Prognostic significance of using solid versus whole tumor size on high-resolution computed tomography for predicting pathologic malignant grade of tumors in clinical stage IA lung adenocarcinoma: a multicenter study. J Thorac Cardiovasc Surg 2012;143:607-12.

3. Asamura H. Minimally invasive approach to early, peripheral adenocarcinoma with ground-glass opacity appearance. Ann Thorac Surg 2008;85:S701-4.

4. Suzuki K, Koike T, Asakawa T, et al. A prospective radiological study of thin-section computed tomography to predict pathological noninvasiveness in peripheral clinical IA lung cancer (Japan Clinical Oncology Group 0201). J Thorac Oncol 2011;6:751-6.

5. Hattori A, Takamochi K, Matsunaga T, et al. Oncological outcomes of sublobar resection for clinical-stage IA high-risk non-small cell lung cancer patients with a radiologically solid appearance on computed tomography. Gen Thorac Cardiovasc Surg 2016;64:18-24.

6. Tsurugai Y, Kozuka T, Ishizuka N, et al. Relationship between the consolidation to maximum tumor diameter ratio and outcomes following stereotactic body radiotherapy for stage I non-small-cell lung cancer. Lung Cancer 2016;92:47-52.

7. Ikeda N, Yoshimura A, Hagiwara M, et al. Three dimensional computed tomography lung modeling is useful in simulation and navigation of lung cancer surgery. Ann Thorac Cardiovasc Surg 2013;19:1-5.

8. Hagiwara M, Shimada Y, Kato Y, et al. High-quality 3-dimensional image simulation for pulmonary lobectomy and segmentectomy: results of preoperative assessment of pulmonary vessels and short-term surgical outcomes in consecutive patients undergoing video-assisted thoracic surgerydagger. Eur J Cardiothorac Surg 2014;46:e120-6.

9. Saji H, Inoue T, Kato Y, et al. Virtual segmentectomy based on high-quality three-dimensional lung modelling from computed tomography images. Interact Cardiovasc Thorac Surg 2013;17:227-32.

10. Furumoto H, Shimada Y, Imai K, et al. Prognostic impact of the integration of volumetric quantification of the solid part of the tumor on 3DCT and FDG-PET imaging in clinical stage IA adenocarcinoma of the lung. Lung Cancer 2018;121:91-6.

11. Makino Y, Shimada Y, Hagiwara M, et al. Assessment of emphysema severity as measured on three-dimensional computed tomography images for predicting respiratory complications after lung surgery. Eur J Cardiothorac Surg 
2018;54:671-6.

12. Goldstraw P, Chansky K, Crowley J, et al. The IASLC Lung Cancer Staging Project: Proposals for Revision of the TNM Stage Groupings in the Forthcoming (Eighth) Edition of the TNM Classification for Lung Cancer. J Thorac Oncol 2016;11:39-51.

13. Maeda R, Yoshida J, Ishii G, et al. Prognostic impact of intratumoral vascular invasion in non-small cell lung cancer patients. Thorax 2010;65:1092-8.

14. Shimada Y, Saji H, Yoshida K, et al. Pathological vascular invasion and tumor differentiation predict cancer recurrence in stage IA non-small-cell lung cancer after complete surgical resection. J Thorac Oncol 2012;7:1263-70.

15. Mimae T, Tsutani Y, Miyata Y, et al. Role of lymphatic invasion in the prognosis of patients with clinical node-negative and pathologic node-positive lung adenocarcinoma. J Thorac Cardiovasc Surg 2014;147:1820-6.

16. Neri S, Yoshida J, Ishii G, et al. Prognostic impact of microscopic vessel invasion and visceral pleural invasion in non-small cell lung cancer: a retrospective analysis of 2657 patients. Ann Surg 2014;260:383-8.

Cite this article as: Shimada Y, Furumoto H, Imai K, Masuno R, Matsubayashi J, Kajiwara N, Ohira T, Ikeda N. Prognostic value of tumor solid-part size and solid-part volume in patients with clinical stage I non-small cell lung cancer. J Thorac Dis 2018;10(12):6491-6500. doi: 10.21037/jtd.2018.11.08
17. Kawase A, Yoshida J, Miyaoka E, et al. Visceral pleural invasion classification in non-small-cell lung cancer in the 7 th edition of the tumor, node, metastasis classification for lung cancer: validation analysis based on a large-scale nationwide database. J Thorac Oncol 2013;8:606-11.

18. Okada M, Nishio W, Sakamoto T, et al. Discrepancy of computed tomographic image between lung and mediastinal windows as a prognostic implication in small lung adenocarcinoma. Ann Thorac Surg 2003;76:1828-32; discussion 1832.

19. Uehara H, Tsutani Y, Okumura S, et al. Prognostic role of positron emission tomography and highresolution computed tomography in clinical stage IA lung adenocarcinoma. Ann Thorac Surg 2013;96:1958-65.

20. Matsuguma H, Yokoi K, Anraku M, et al. Proportion of ground-glass opacity on high-resolution computed tomography in clinical T1 N0 M0 adenocarcinoma of the lung: A predictor of lymph node metastasis. J Thorac Cardiovasc Surg 2002;124:278-84.

21. Takenaka T, Yamazaki K, Miura N, et al. The Prognostic Impact of Tumor Volume in Patients with Clinical Stage IA Non-Small Cell Lung Cancer. J Thorac Oncol 2016;11:1074-80. 